\title{
Imaging performance of phase-contrast breast computed tomography with synchrotron radiation and a CdTe photon- counting detector
}

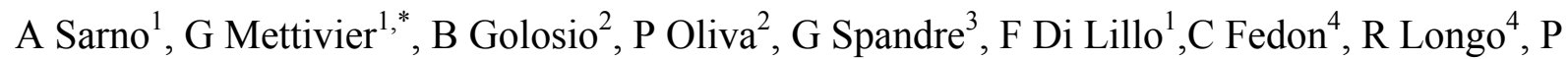 \\ Russo $^{1}$ \\ ${ }^{1}$ Universita' di Napoli Federico II, Dipartimento di Fisica "Ettore Pancini", and INFN Sezione di Napoli, Napoli, \\ Italy \\ ${ }^{2}$ Universita' di Sassari, Sassari, Italy, and INFN Sezione di Cagliari, Cagliari, Italy \\ ${ }^{3}$ INFN Sezione di Pisa, Pisa, Italy \\ ${ }^{4}$ Universita' di Trieste, Dipartimento di Fisica, and INFN Sezione di Trieste, Trieste, Italy
}

\begin{abstract}
Purpose: Within the SYRMA-CT collaboration based at the ELETTRA synchrotron radiation (SR) facility (Trieste, Italy) the authors investigated the imaging performance of the phase-contrast computed tomography (CT) system dedicated to monochromatic in vivo 3D imaging of the female breast, for breast cancer diagnosis.

Methods: Test objects were imaged at $38 \mathrm{keV}$ using monochromatic SR and a high-resolution CdTe photon-counting detector. Signal and noise performance were evaluated using modulation transfer function (MTF) and Noise Power Spectrum (NPS). Phase-contrast CT images as well as images obtained after the application of a phase-retrieval algorithm were evaluated. The contrast to noise ratio $(\mathrm{CNR})$ and the capability of detecting test microcalcification clusters and soft masses were explored.

Results: For a voxel size of $(60 \mu \mathrm{m})^{3}$, phase-contrast images showed higher spatial resolution $(6.7$ $\mathrm{mm}^{-1}$ at $\left.10 \% \mathrm{MTF}\right)$ than corresponding phase retrieval images $\left(2.5 \mathrm{~mm}^{-1}\right)$. Phase retrieval produced a reduction of the noise level as well as an increase of the CNR by more than one order of magnitude, compared to raw phase-contrast images. $\mathrm{CaCO}_{3}$ microcalcifications with a diameter down to $130 \mu \mathrm{m}$ were detected both in phase-contrast and in phase retrieval images of the test object.

Conclusions: The investigation on test objects indicates that breast CT with a monochromatic SR source is technically feasible in terms of spatial resolution, image noise and contrast, for in vivo 3D imaging with a dose comparable to that of two-view mammography. Phase-retrieved images showed the best performance in the trade-off between spatial resolution and image noise.
\end{abstract}

Keywords: Synchrotron radiation; breast cancer; photon-counting detector; computed tomography; phase-contrast imaging

* Corresponding author: mettivier@na.infn.it 


\section{Introduction}

In the fight against breast cancer, mammography is the gold standard technique for screening and plays a fundamental role in diagnostic exams. However, the performance of 2D mammography for breast cancer detection is not ideal. The Breast Cancer Surveillance Consortium (BCSC), by means of performance assessment of screening mammography examinations $(1,838,372$ exams in the period 2004-2008), determined a sensitivity of $84.4 \%$ and a specificity of $90.8 \%$ [1]. A reduced diagnostic performance in detecting tumor masses can be due to the presence of overlying normal breast tissue that can obscure breast lesions and hinder their detection, producing a wrong diagnosis. In order to improve breast cancer detection via 3D X-ray imaging, computed tomography (CT) scanners dedicated to the breast (Breast CT, or BCT) have been developed, since 2001, as reviewed recently [2].

Moreover, phase-contrast mammography [3-5] and phase-contrast breast tomography [6-14] are under investigation as new imaging techniques for a potentially better definition and increased conspicuity of breast lesions at diagnosis. This potential is related to the increased tissue contrast produced by coherent X-ray imaging, either with monochromatic synchrotron radiation (SR) X-ray sources $[9,15]$ or with polychromatic X-ray tube sources and phase detection techniques $[7,16]$. In this context, BCT using synchrotron radiation (SR-BCT) may play a relevant role, both as a reference imaging technique and in order to exploit opportunities offered by a tunable, monochromatic X-ray source with extremely high spatial (lateral) coherence and high photon flux, in a parallel beam geometry. Research groups at the SR facility ELETTRA (Trieste, Italy) [3-5,17-19], at the European Synchrotron Radiation Facility in Grenoble, France [6-9] and at the Imaging and Medical beamline of the Australian Synchrotron [20] investigated these phase-contrast based breast imaging techniques. In particular, recent results [18-21] showed that the in-line (propagation based) phase-contrast technique offers significant improvement in image quality compared to conventional absorption-based BCT. This is a significant indication, since in the field of phase-contrast imaging free-space propagation based techniques offer advantages of simplicity, reliability and feasibility of clinical implementation, with respect to other techniques requiring complex optical setups.

In this line of research, the SYRMA-CT collaboration, based at the ELETTRA SR facility, investigates the technique of phase-contrast in vivo SR tomography dedicated to the diagnosis of breast cancer, exploiting the propagation based phase-contrast technique, with a laminar and monochromatic SR X-ray source, and a large-area single-photon counting detector [18,21].

The SYRMA-CT acquisition setup, recently upgraded for breast CT acquisitions from previous in vivo planar imaging studies [3], includes a rotating and vertically translating bed where 
the patient is in the prone position with her uncompressed breast freely hanging through a hole in the bed. A first study conducted with a breast specimen has indicated the feasibility of a clinical BCT with SR source [18], and the related impact on breast dosimetry has been investigated recently [21].

The aim of this work is to characterize this SYRMA-CT system for in vivo imaging (via linear systems analysis) in terms of the spatial resolution of the system via determination of its modulation transfer function (MTF) and noise power spectrum (NPS), and by evaluating, on test object, the contrast to noise ratio $(\mathrm{CNR})$ and the visibility of simulated microcalcifications. The analysis focuses on the features of the phase signal on the CT slices, by comparing the imaging performance obtainable in phase-contrast imaging (obtained without phase-retrieval algorithm, but by reconstructing CT slices from the raw flat-field corrected projections), to that obtainable via a phase retrieval algorithm (referred below as phase retrieval imaging or phase-retrieved images).

\section{Materials and methods}

\subsection{Experimental Setup}

The SYRMA-CT project is ongoing at the SYRMEP beamline at ELETTRA [18], a line dedicated to medical physics applications. For the execution of CT acquisitions, the setup was modified compared to the previous one used for phase-contrast mammography [5], in order to perform the rotation (in several turns) of the patient support, with the subject in the prone position and the breast hanging at the rotation center (fig. 1). The radiation source was a bending magnet of the ELETTRA storage ring; the beam energy was selected by means of a $\mathrm{Si}(111)$ double crystal monochromator, which permitted the tuning of the beam energy in the range $8.5-40 \mathrm{keV}$, with an energy resolution of $0.2 \%$. The beam shape in the radiology hutch was defined by a tungsten slits system, as a rectangle of $200 \mathrm{~mm}$ (horizontal width) and a height of $3 \mathrm{~mm}$ (vertical height). The Xray beam transverse coherence length is of the order of $10 \mu \mathrm{m}$ at $15 \mathrm{keV}$ [40]. The CT views were acquired in a simplified setup using a rotation stage in the breast position of the radiology hutch, at a distance of $2 \mathrm{~m}$ from the imaging detector. Mittone et al. [21bis], in a simulation study performed with monochromatic X-ray radiation, showed that the ratio between the dose to the breast and transmittance through the imaged object decreases as the photon energy increases up to about 60 $\mathrm{keV}$. Based on this result, the beam photon energy chosen for the SYRMA-CT project is $38 \mathrm{keV}$, the highest photon energy disposable at ELETTRA without affecting drastically the X-ray beam stability. The projections were collected over $180 \mathrm{deg}$, thanks to the negligible horizontal divergence of the beam ( $7 \mathrm{mrad})$ which approximates a parallel beam geometry. The number of acquired projections was 720 . 


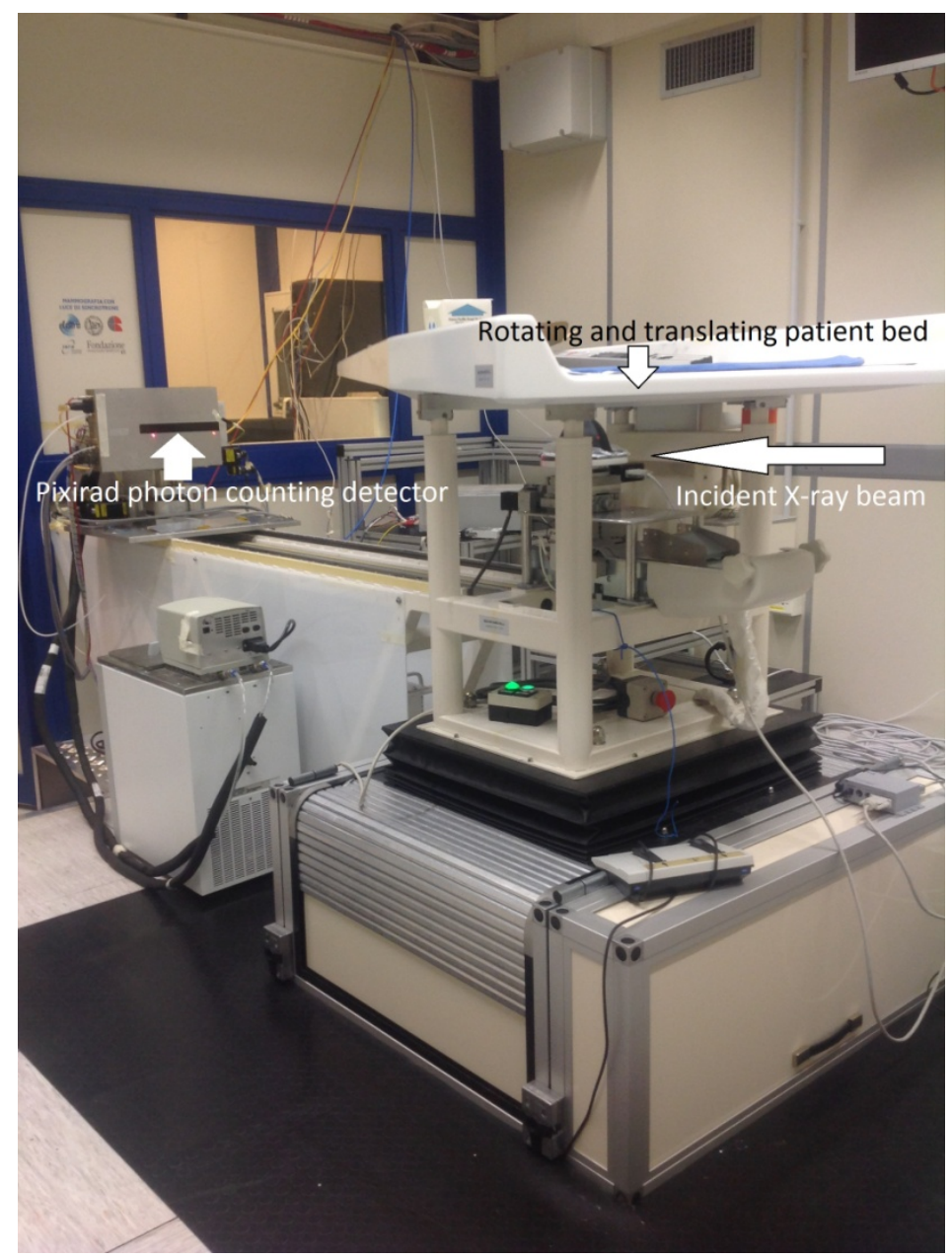

Figure 1: Photo of the SYRMA-CT setup for phase-contrast breast CT at the ELETTRA synchrotron radiation laboratory (Trieste, Italy). The horizontal beam irradiates the breast hanging from a hole in the patient bed; the transmitted beam is recorded by a high resolution photon counting detector. Rotation and translation of the bed permits to acquire in successive axial scan over $180 \mathrm{deg}$ a complete dataset for CT reconstruction.

The detector was PIXIRAD-8, a high efficiency, direct detection, photon counting X-ray imaging detector. This semiconductor pixel detector showed an isotropic spatial resolution of $72 \mu \mathrm{m}$ (full width at half maximum, FWHM, of the detector point spread function). PIXIRAD-8 is a multiblock, 2 Mpixel detector consisting of eight PIXIRAD detector units aligned in a row, produced by PIXIRAD Imaging Counters srl (http://www.pixirad.com), an INFN Pisa spin-off company. The basic block is a hybrid architecture in which the sensor and readout electronics (ASIC) are coupled with the flip-chip bump-bonding technique. For a single unit, the sensor (ACRORAD Co., Ltd.) is a cadmium telluride (CdTe) Schottky type array of diodes with a total area of $30.96 \mathrm{~mm} \times 24.98 \mathrm{~mm}$ and a substrate thickness of $0.650 \mathrm{~mm}$. The pixel arrangement is on a hexagonal honeycomb matrix with a pitch of $60 \mu \mathrm{m}$. The multi block module used in this project is an 8-unit system reaching a total active area of $250 \mathrm{~mm} \times 25 \mathrm{~mm}$; raw images have a size of $4608 \times 476$ pixels. Due to the modular structure of the device, a dead space is present between adjacent blocks, with a width of 
$180 \mu \mathrm{m}$. As outlined in ref. [22], a high Detective Quantum Efficiency of a thick, direct conversion photon-counting detector (1-mm thick CdTe sensor in their study) may allow for a dose reduction with respect to energy-integrating scintillator-based detectors used in current BCT setups with polychromatic sources and cone-beam geometry.

The CT scans were performed in step-and-shoot modality, with an exposure time of $50 \mathrm{~ms}$ per view. This implies that in this work, the system spatial resolution and noise are not affected by the blurring introduced by the continuous rotation of the object.

A low-level discriminator threshold equivalent to $19 \mathrm{keV}$ (corresponding to half the energy of the incident photons) was set on the pixel signal; this permitted minimizing charge-sharing effects in the pixel signal (double counts, loss of spatial resolution) thus avoiding a loss of counts [23]. This high-value threshold makes the dark noise of the detector negligible [24]. The images were flat-field corrected to apply gain correction. Subsequently, a re-sampling of the images based on linear interpolation was performed, in order to change from a honeycomb to a square pixel array, producing images with $30-\mu \mathrm{m}$ equivalent pixel pitch.

The reconstructed slices were obtained from the pre-processed projections using Filtered Back Projection (FBP). In order to reconstruct slices with voxel size of $(60 \mu \mathrm{m})^{3}$ and $(120 \mu \mathrm{m})^{3}$, projections underwent a $2 \times 2$ binning and a $4 \times 4$ binning process respectively. The phase signal was retrieved by processing the projections with the algorithm proposed by Paganin et al [25]. In order to implement this algorithm, the scanned objects are assumed to be composed of one homogeneous material, with known real part $(1-\delta)$ and imaginary part $(\beta)$ of the complex refractive index of the material, $n(E)=(1-\delta)+i \beta$, evaluated at the photon energy $E$.

\subsection{System MTF}

The high-resolution detector of the SYRMA-CT system, and the practical absence of penumbra due to the almost parallel beam geometry ( $7 \mathrm{mrad}$ beam divergence), contribute to produce high-resolution CT scans over a 180-deg angular span. The spatial resolution of the SYRMA-CT scanner was evaluated by measuring the system MTF; a limiting spatial frequency was evaluated at $10 \% \mathrm{MTF}$ value $\left(\mathrm{MTF}_{0.1}\right)$. A sharp polymethylmethacrylate (PMMA) edge (for which $\delta(E)=1.845 \times 10^{-7}$ and $\beta(E)=1.219 \times 10^{-11} @ E=38 \mathrm{keV}$; thickness =2 mm; height = $\left.90 \mathrm{~mm}\right)$ [26] was imaged in order to evaluate the system edge spread function (ESF), from which the line spread function (LSF) of the system was evaluated, by numerical differentiation. In order to reduce the noise, in the case of the MTF evaluation over the PMMA edge, the ESF curves have been averaged over 10 consecutives rows. The PMMA slab introduced little attenuation but sizeable phase shifts in the X-ray beam. Then, the system MTF (normalized to the value at zero spatial frequency) was computed via numerical Fourier transform. It is worth noting that the fringes on the phase-contrast 
image of the PMMA edge (determining the edge enhancement effect typically observed in in-line phase-contrast imaging) may distort the MTF curves derived in the above way, introducing an artifact (i.e. normalized MTF values greater than 1) similar to the one occurring in CT reconstruction with the use of some kernels, characterized by undershoots in the kernel curve (see, e.g., [27]).

The system MTF was evaluated also by using a highly attenuating object, a $50-\mu \mathrm{m}$ diameter tungsten wire placed parallel to rotation axis, at $50 \mathrm{~mm}$ radial distance from the axis, slightly tilted (2 deg) with respect to the rotation axis in order to obtain the presampled LSF. This technique is commonly used for spatial resolution assessment in cone-beam breast CT [28,29]. The evaluation of the system MTF was carried out with two reconstruction voxel sizes $\left(60 \times 60 \times 60 \mu^{3}\right.$ and $\left.120 \times 120 \times 120 \mu \mathrm{m}^{3}\right)$.

\subsection{System NPS}

The common metric for the evaluation of noise properties in attenuation based CT imaging is the (3D) NPS, which describes the spatial decomposition of noise variance in an image as a function of the spatial frequency, thus expressing the noise transfer property of the system. Assuming the applicability of linear analysis system theory for phase-contrast imaging, for the experimental determination of the NPS a scan was performed of a homogeneous mammographic phantom $\left(\delta(E)=1.619 \times 10^{-7} ; \beta(E)=0.697 \times 10^{-11} ; @ E=38 \mathrm{keV}\right.$ [30]), a CIRS phantom BR 50/50 mod. $014 \mathrm{AD}$ simulating a 50/50 breast tissue (i.e. a 50\% glandular, 50\% adipose tissue) (further details are reported in sec 2.4.). The $1 \mathrm{D}$ coronal NPS was evaluated as indicated in ref. [31]. The 2D NPS was estimated by the 2D fast Fourier transform from a region of interest (ROI), which did not include any targets, in the coronal view. Then, the 1D noise power spectrum was computed by radially averaging the 2D NPS. Pixel values in phase-contrast CT slices represent the attenuation coefficient (proportional to the local value of $\beta$ ) of the imaged object, and the pixel value of images after phase-retrieval is proportional to $\delta$. Then, normalized NPS (NNPS) curves were evaluated, by dividing the NPS curves for the square root of the mean signal value in the ROI where the NPS was evaluated. These curves do not depend on the signal units of measurements, so permitting to compare noise power spectrum in phase-contrast to that in phase retrieval imaging.

\subsection{Contrast and microcalcification visibility}

In order to test the microcalcification visibility and CNR in tomographic images, the multilayer commercial test object (CIRS mod. 014AD) was imaged. Figure 2 shows a radiography of this object (total thickness $=85 \mathrm{~mm}$ ), obtained via a mammography unit (Siemens Mammomat 
Inspiration) at $32 \mathrm{kVp}(\mathrm{W} / \mathrm{Rh})$, with focal spot of $0.3 \mathrm{~mm}$, pixel size of $85 \mu \mathrm{m}$ and entrance air kerma of $15.35 \mathrm{mGy}$. The test object comprises several layers simulating the attenuation coefficient of a breast tissue with an average glandular fraction of 50\%; one layer contains different targets for image quality analysis. Its target layer includes clusters of alumina and $\mathrm{CaCO}_{3}$ grains simulating microcalcifications (with diameters from 130 to $390 \mu \mathrm{m}$ ), four high-contrast fibers with a diameter of $25 \mu \mathrm{m}$, six masses (hemispheres with diameter in the range 1.8-6.32 $\mathrm{mm}$, simulating $75 \%$ glandular tissue attenuation) and a 5-mm thick contrast single step-wedge (adipose/glandular tissue). Just one rectangular slab $\left(20\right.$-mm thick with a size of $\left.100 \times 125 \mathrm{~mm}^{2}\right)$ of the multilayer phantom was scanned with the SR setup.

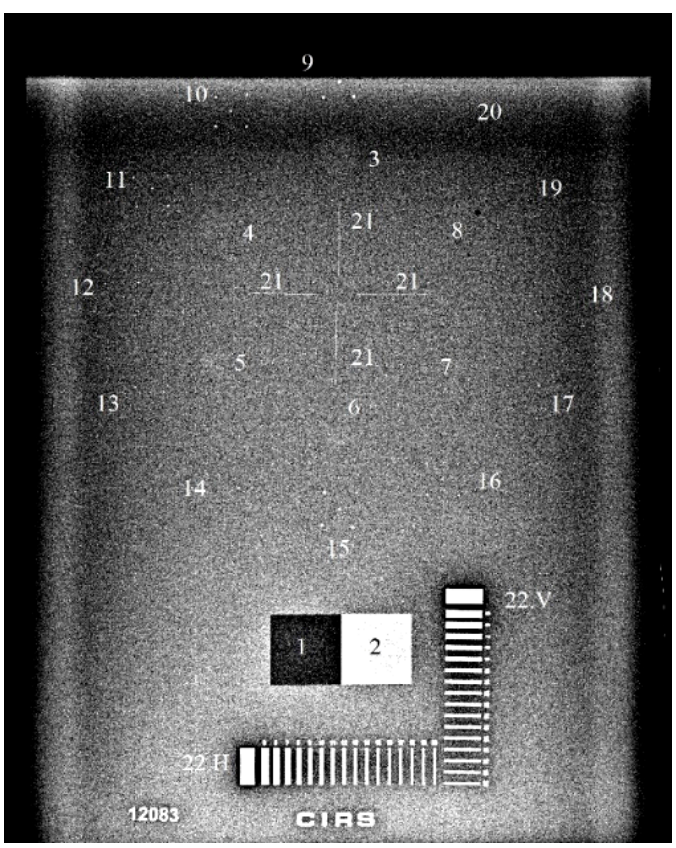

Figure 2: Radiography along cranio-caudal direction of the CIRS test object BR50/50 mod. 014AD (50\% glandular breast tissue attenuation), shown for the purpose of object description. It includes a contrast step-wedge target (labels 1 and 2, superimposed on the image, for adipose and glandular tissue, respectively), six 75\% glandular hemispheric masses (labels 3-7) with a radius in the range 3.16-0.90 mm, clusters of alumina (labels 15-20) and $\mathrm{CaCO}_{3}$ (labels 9-14) (from 390 to $130 \mu \mathrm{m}$ ) microcalcifications, high-contrast fibers with a diameter of $25 \mu \mathrm{m}$ (label 21) and bar patterns (labels 22.H and 22.V). Setup: Siemens Mammomat Inspiration, $32 \mathrm{kVp}$ (W/Rh), focal spot size $0.3 \mathrm{~mm}$, detector pixel size $85 \mu \mathrm{m}$, entrance air kerma $15.35 \mathrm{mGy}$.

The image CNR was evaluted as:

$$
\mathrm{CNR}=\sqrt{\mathrm{A}} \frac{\left|\mathrm{S}_{\mathrm{a}}-\mathrm{S}_{\mathrm{b}}\right|}{\sqrt{\frac{\sigma_{\mathrm{a}}^{2}+\sigma_{\mathrm{b}}^{2}}{2}}}
$$

where $S_{\mathrm{a}}$ and $S_{\mathrm{b}}$ are the average pixel values in the selected ROIs, respectively, $\sigma^{2}{ }_{\mathrm{a}}$ and $\sigma_{\mathrm{b}}^{2}$ are the corresponding variances and $A$ is the area of the ROI (in pixels).

Two ROIs each of $100 \times 100$ pixels were selected in the step-wedge inclusion of the test object (one for fat and one for glandular materials, respectively): in this specific case, $S_{\mathrm{a}}$ and $\sigma_{\mathrm{a}}^{2}$ are 
the signal and the variance in the glandular inclusion and $S_{\mathrm{b}}$ and $\sigma^{2}{ }_{\mathrm{b}}$ for the signal and the variance in the fat inclusion, respectively.

A global figure of merit $(Q)$ was evaluated in order to compare the imaging performance of the SR system in phase-contrast imaging to that obtainable with phase-retrieval. This index was proposed in ref. [32] to obtain a single figure of merit in CT images taking into account the system spatial resolution, the image noise $\left(\sigma^{2}\right)$ and the radiation dose to the tissue $(D)$ :

$$
Q=c \frac{\mathrm{MTF}_{0.1}^{4}}{\sigma^{2} \cdot D}
$$

where $c$ is a constant. $Q$ takes high values when the spatial resolution is high, the signal noise is low and the dose is low. Since the compared images were obtained with the same amount of radiation dose, the quantity $c / D$ was fixed to $1 \mathrm{~mm}^{4}$. The noise was evaluated as the variance in the ROI selected for the NPS evaluation, normalized to the square root of the mean signal value in the same region.

\subsection{Dose assessment}

A MC simulation was developed for the mean glandular dose estimation in this experiment [21,36]. A dosimetric formalism for the estimate of the glandular dose in a SR-BCT exam with monochromatic radiation has been defined [21]. Since irradiation of the pendant breast with a thin laminar SR beam implies the successive axial scan of adjacent thin sections of the breast along the vertical direction, two dose metrics were introduced, namely, the mean glandular dose in the irradiated volume $\left(\mathrm{MGD}_{\mathrm{v}}\right)$ and the total mean glandular dose $\left(\mathrm{MGD}_{\mathrm{t}}\right)$. The latter quantity takes into account the energy deposited by radiation scattered outside the irradiated volume, by attributing this additional dose term to the glandular tissue in the irradiated volume. A mean dose to the glandular breast tissue in the case of SR-BCT irradiation of the whole breast (MGD) was also calculated, for direct comparison with the mean glandular dose in a mammography or in a cone-beam BCT scan with dedicated scanners. The three dose metrics can be calculated from determinations of the corresponding normalized glandular dose coefficients (reported in [21] from 8 to $50 \mathrm{keV}$ in $1-\mathrm{keV}$ increments, for $0 \%, 50 \%$ and $100 \%$ glandular fraction by weight and for cylindrical model breasts of diameter from 8 to $16 \mathrm{~cm}$ ) and multiplication by the air kerma free-in-air at isocenter.

In this work, the air kerma at the isocenter during the phantom scanning was $10.4 \mathrm{mGy}$ both for the NNPS assessment and for the test object imaging, with a 3-mm beam height. The corresponding $\mathrm{MGD}_{\mathrm{v}}$ value was $4.2 \mathrm{mGy}$ and the $\mathrm{MGD}_{\mathrm{t}}$ was $8.0 \mathrm{mGy}$, calculated by approximating the test object as a cylindrical breast with an equivalent diameter of $12 \mathrm{~cm}$ and a height of $9 \mathrm{~cm}$, and 
$50 \%$ glandular fraction (since the test object had an height of just $2 \mathrm{~cm}$, the value given for the $\mathrm{MGD}_{\mathrm{t}}$ is only an upper limit to the $\mathrm{MGD}_{\mathrm{t}}$ value for the actual irradiated section). The difference in these values is the ratio of the scattered energy imparted to the not-directly irradiated volume, to the glandular mass in the irradiated volume. In assessing the mean glandular dose for future in vivo BCT scans with a monochromatic SR beam of 3-mm height, for a 12-cm breast size of 9-cm height fully irradiated in successive axial scans from the chest wall to the nipple, the authors estimate a value of about $7.3 \mathrm{mGy}$ for the MGD to the whole breast with data in ref. [21] and for an air kerma at isocenter of $10.4 \mathrm{mGy}$, at $38 \mathrm{keV}$. Such a value is equivalent to the MGD used in two-view mammography, and it will be the one used in the programmed clinical trial.

\section{Results}

\subsection{MTF}

Figure 3 shows the ESF curves in phase-contrast (fig. 3a) and in phase retrieval (fig. 3b), respectively, for a PMMA-air edge. The edge profile in phase-contrast shows fringes (fig. 3a) due to the refraction of the X-ray beam; as expected, this phenomenon is not visible after the application of the phase retrieval algorithm on the projections (fig. 3b). The differentiation of the ESF curves produces the LSF curves in fig. 4; the phase effects in the phase-contrast ESF cause dips in the corresponding LSF (fig. 4a), at variance with phase retrieval LSF (fig. 4b). The high attenuation coefficient of the tungsten wire makes the phase effects less relevant: indeed, fringes are not visible in the presampled LSF evaluated from the wire image (fig. 5).
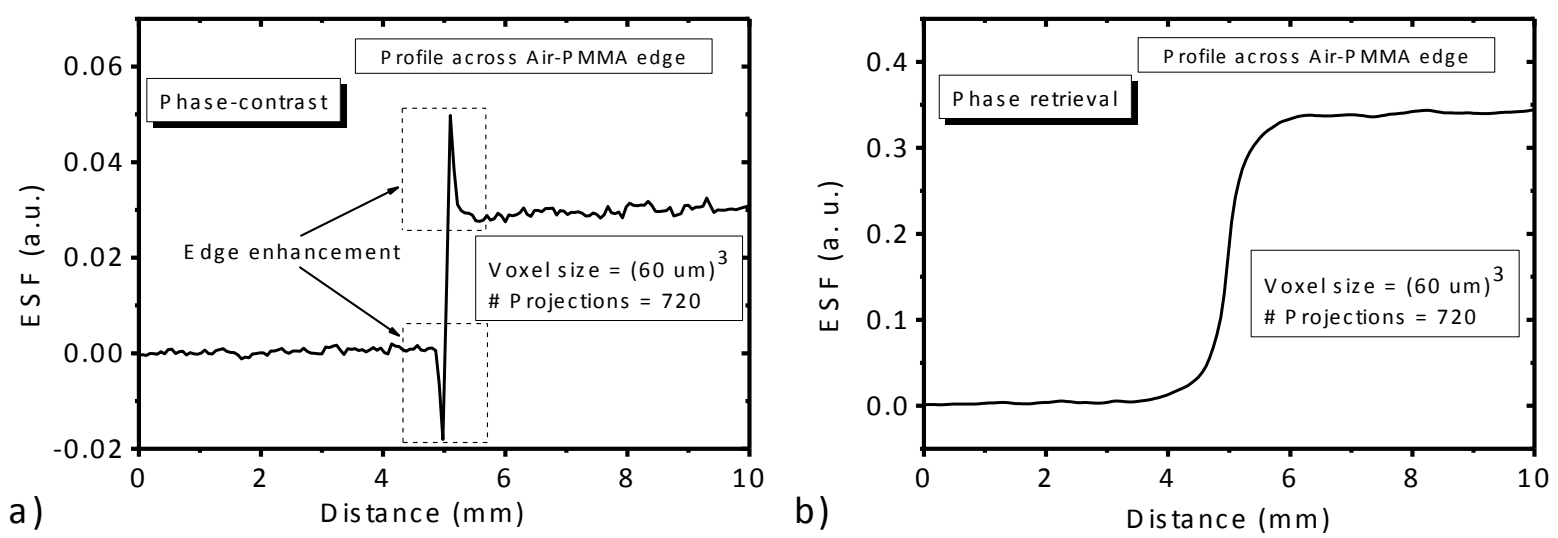

Figure 3: The ESFs across a sharp PMMA-air edge in phase-contrast imaging (a) and with phase-retrieval (b) averaged over 60 rows. In phase-contrast imaging, the fringes due to phase effects are outlined. Voxel size $=(60 \mu \mathrm{m})^{3}$; number of projections $=720$.

Figure 6 shows the system MTF both in phase-contrast (fig. 6a) and in phase retrieval (fig. $6 b)$, evaluated using the PMMA edge. The curves were obtained from 720 projections equally spaced over a 180-deg scan and with an isotropic voxel of $(60 \mu \mathrm{m})^{3}$ and of $(120 \mu \mathrm{m})^{3}$. The edge effects cause an increase in the signal, as demonstrated (fig. 6a). 

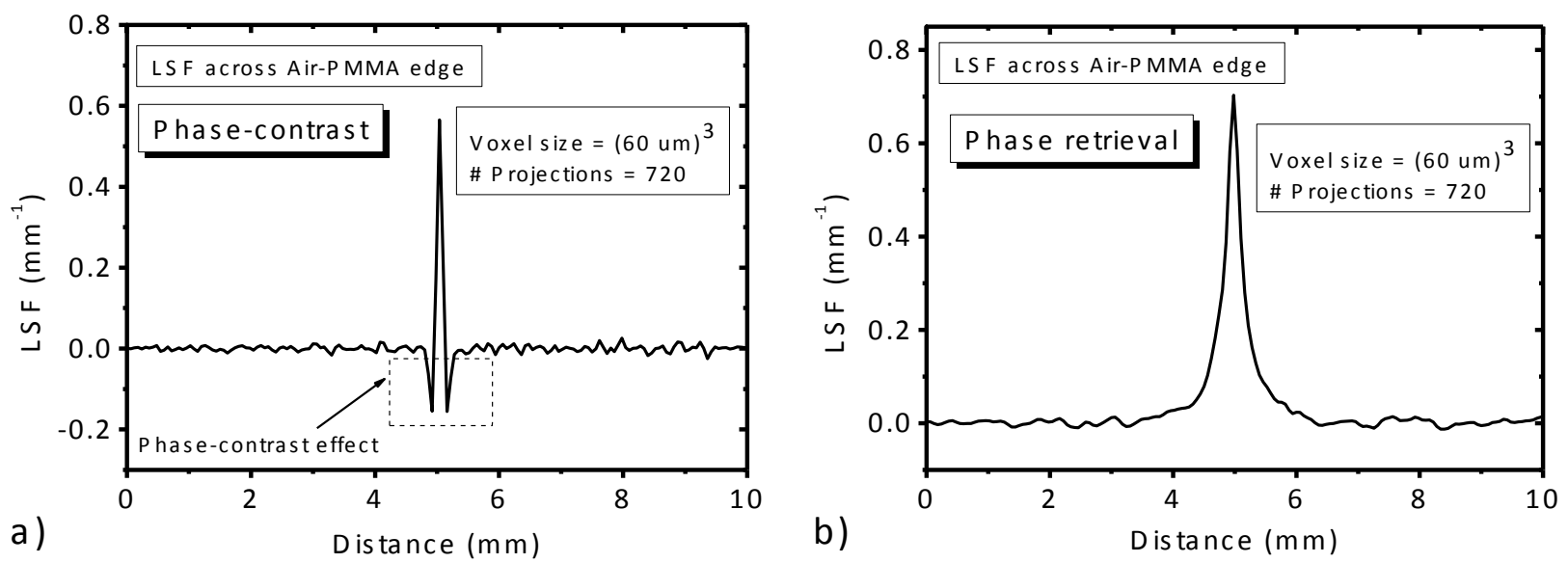

Figure 4: The LSFs in phase-contrast (a) and after phase retrieval (b) obtained by differentiating the ESF curves in fig. 3. The phase-contrast fringes in the ESF in phase-contrast imaging cause dip signals in LSF curve. Voxel size $=(60$ $\mu \mathrm{m})^{3}$; number of projections $=720$.

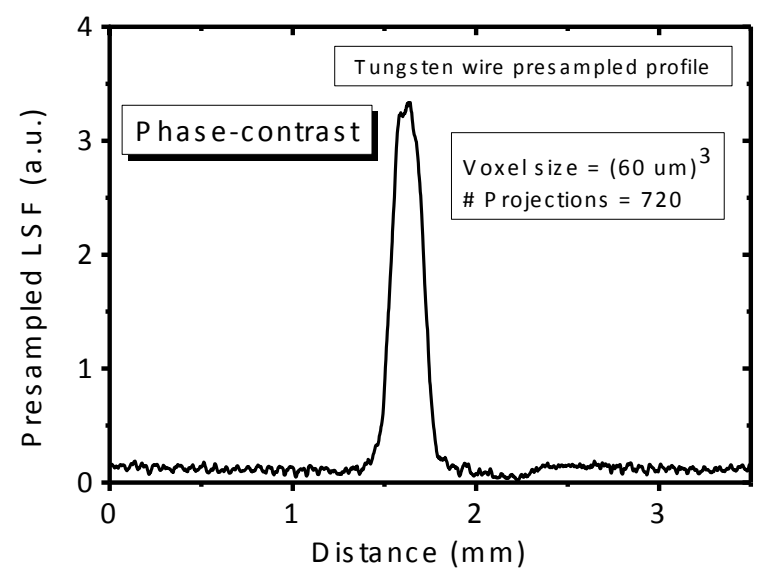

Figure 5: The presampled LSF in phase-contrast imaging evaluated with a $50-\mu \mathrm{m}$ diameter tungsten wire. Voxel size $=$ $(60 \mu \mathrm{m})^{3} ;$ number of projections $=720$.
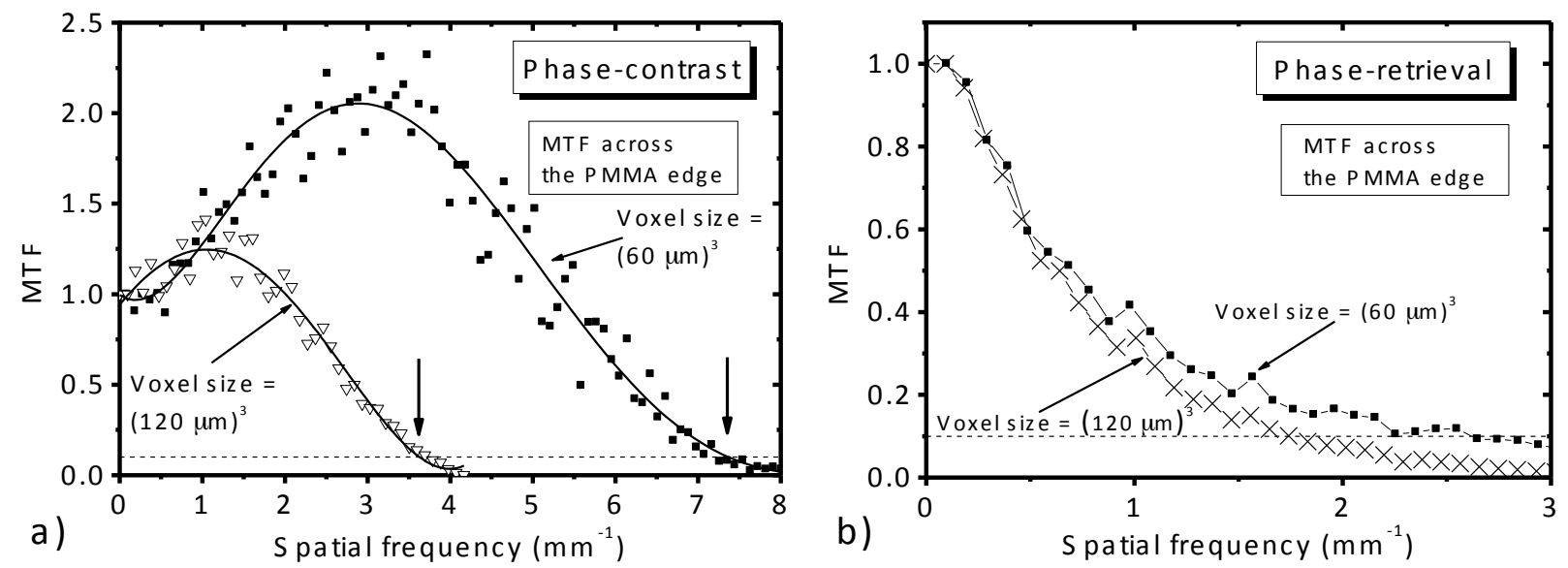

Figure 6: The MTF curves in phase-contrast (a) and in phase-retrieval imaging (b), evaluated across the PMMA edge, with voxel size of $(60 \mu \mathrm{m})^{3}$ and $(120 \mu \mathrm{m})^{3}$ obtained with 720 projections equally spaced over 180 deg rotation scan. The dashed horizontal line indicates the $10 \%$ MTF value.

The presence of edge-enhancement effects determine MTF curves which exceed the normalization value at zero spatial frequency. Due to the system spatial resolution reduction, such a 
phenomenon reduces as the voxel size increases from $(60 \mu \mathrm{m})^{3}$ to $(120 \mu \mathrm{m})^{3}$. Honda et al. [33] showed a similar result in polychromatic propagation based phase-contrast mammography, related to an "improvement of sharpness caused by the edge effect" [33].

Figure 7 shows system MTF curves in phase-contrast imaging (evaluated with the method of the thin tungsten wire) not influenced by the phase-contrast effects. In these curves the limiting frequency at $\mathrm{MTF}_{0.1}$ is $6.7 \mathrm{~mm}^{-1}$ with voxel of $(60 \mu \mathrm{m})^{3}$; it reduces to $2.5 \mathrm{~mm}^{-1}$ when a Paganin filter was applied over the projections (fig. 6b). Increasing the voxel size to $(120 \mu \mathrm{m})^{3}$ reduces the limiting spatial frequency in phase-contrast (evaluated with the tungsten wire) and in phase retrieval (evaluated over the PMMA edge) down to $4 \mathrm{~mm}^{-1}$ and $1.7 \mathrm{~mm}^{-1}$, respectively. However, the frequency at $\mathrm{MTF}_{0.1}$ evaluated with the method of the thin tungsten wire $\left(6.7 \mathrm{~mm}^{-1}\right.$ for a voxel size of $\left.(60 \mu \mathrm{m})^{3}\right)$ does not differ significantly from that obtained with PMMA edge $7.2 \mathrm{~mm}^{-1}$ ); for a voxel size of $(120 \mu \mathrm{m})^{3}$ the limiting frequency are $4 \mathrm{~mm}^{-1}$ and $3.8 \mathrm{~mm}^{-1}$, respectively.

Figure 7bis shows the MTF curves over a tungsten wire, in phase-contrast imaging, at $10 \mathrm{~mm}$, $30 \mathrm{~mm}$ and $50 \mathrm{~mm}$ from isocenter. The curves do not significantly differ each other, and the slight difference can be attributed to the uncertainty in the evaluation.

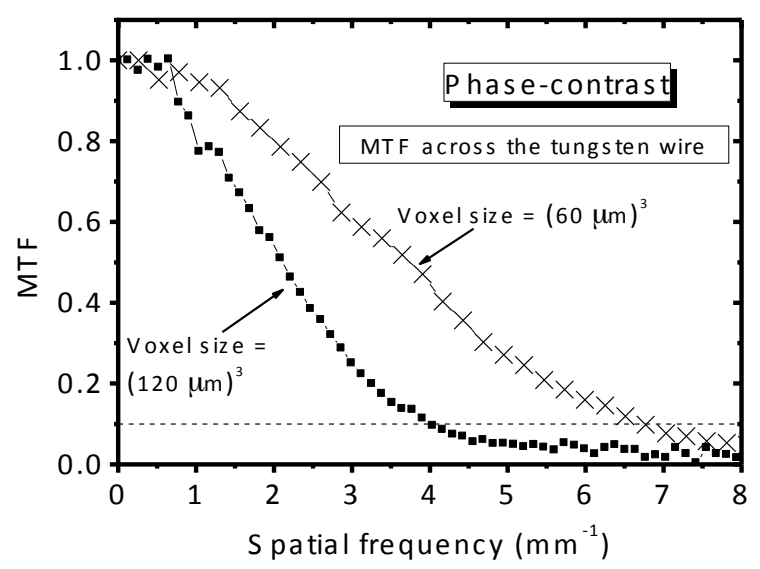

Figure 7: The MTF curves in phase-contrast imaging, evaluated over the thin tungsten wire, with voxel size of (60 $\mu \mathrm{m})^{3}$ and $(120 \mu \mathrm{m})^{3}$ and with 720 projections equally spaced over $180 \mathrm{deg}$ rotation scan. The dashed lines indicates the $10 \%$ MTF value. 


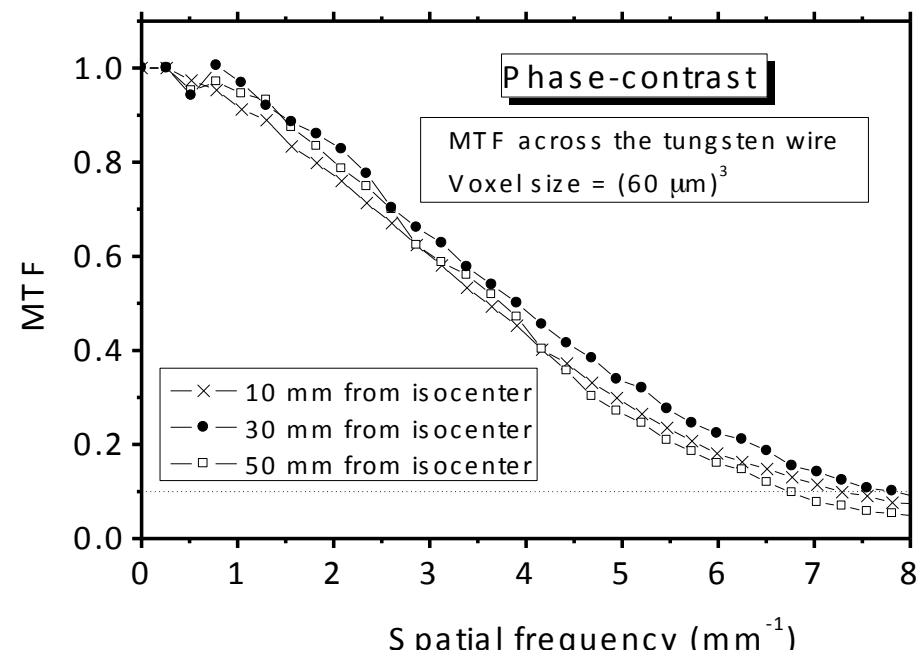

Figure 7bis: The MTF curves in phase-contrast imaging, evaluated over the thin tungsten wire, with voxel size of $(60$ $\mu \mathrm{m})^{3}$ and 720 projections equally spaced over $180 \mathrm{deg}$ rotation scan at $10 \mathrm{~mm}, 30 \mathrm{~mm}$ and $50 \mathrm{~mm}$ from the scanner isocenter.

\subsection{NNPS}

Figure 8 shows the NNPS curves obtained in phase-contrast imaging (fig. 8a) and in phase retrieval (fig. 8b). Such curves were evaluated with an image voxel size of $(60 \mu \mathrm{m})^{3}$ as well as for a voxel of $(120 \mu \mathrm{m})^{3}$. Since the (raw processed) detector pixel pitch is $30 \mu \mathrm{m}$, the corresponding signal bandwidth extends up to $16.7 \mathrm{~mm}^{-1}$ [31], and in the case of reconstructed coronal pixel sizes of $60 \mu \mathrm{m}$ and $120 \mu \mathrm{m}$, the sampling theorem indicates that signal aliasing may be present, producing signal spectra distortions. This effect was observed in the NNPS curves in phase-contrast imaging, where the signal deviates significantly from zero for the highest frequencies (fig. 8a). The Paganin filter used for the phase retrieval is a low-pass filter, which decreases signal noise as well as the influence of aliasing (fig. 8b). The voxel size presents strong influence on NNPS in phasecontrast: indeed, the maximum of the curve with voxel size of $(120 \mu \mathrm{m})^{3}$ is less than half of the maximum of the curve related to a voxel size of $(60 \mu \mathrm{m})^{3}$. The voxel size had weaker influence on NNPS obtained with phase retrieval. The NNPS curves with phase retrieval show a drastic reduction in the noise level in comparison to that in images obtained without phase retrieval. For a voxel size of $(60 \mu \mathrm{m})^{3}$, the maximum of the NNPS curve in phase-contrast images is more than one order of magnitude greater than the maximum of the curve with phase retrieval. Moreover, the first curve presents a maximum at $3 \mathrm{~mm}^{-1}$, while the NNPS in images with phase retrieval has its maximum at about $1 \mathrm{~mm}^{-1}$. The noise in phase retrieval imaging, being less prominent at high spatial frequencies than in the case of phase-contrast imaging, may better fit the need of detecting smaller lesions, and balance the reduction in the system spatial resolution due to the Paganin filtering. 

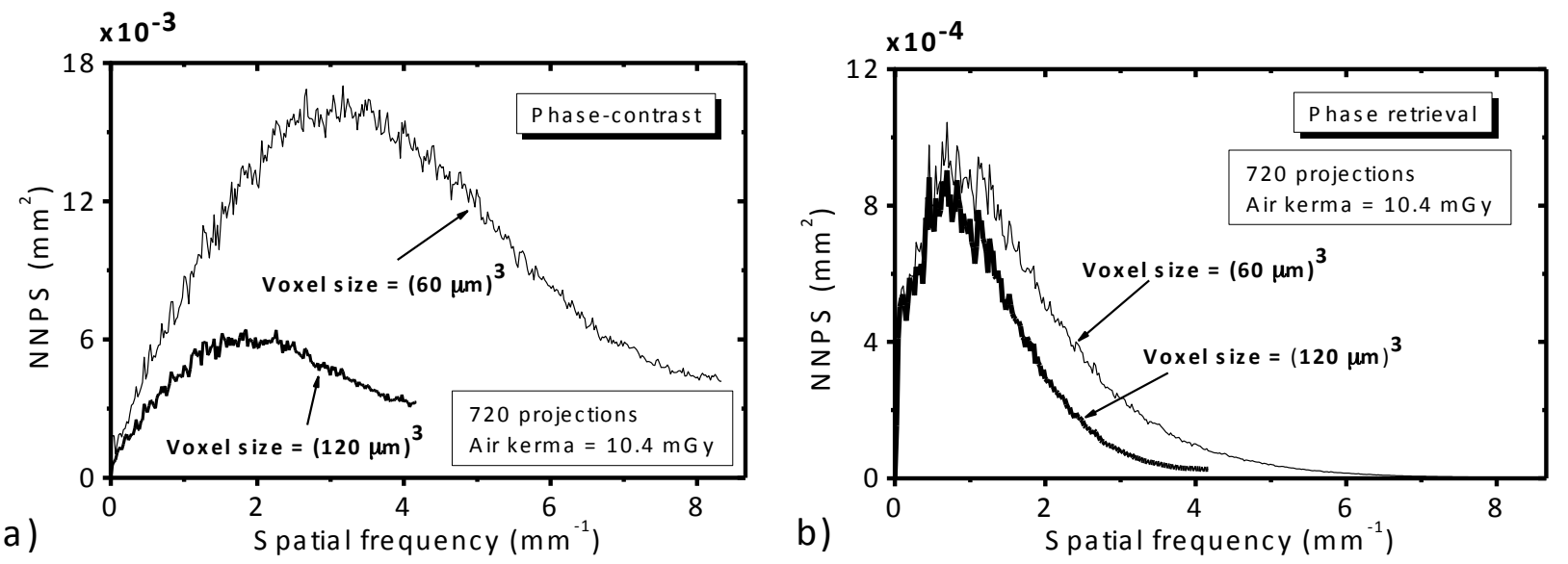

Figure 8: NNPS in phase-contrast imaging (a) and with phase-retrieval (b). They were evaluated both with a voxel size of $(60 \mu \mathrm{m})^{3}$ and of $(120 \mu \mathrm{m})^{3}$ and the reconstructions were performed from 720 projections equally spaced over a 180 deg rotation scan. Air kerma at isocenter $=10.4 \mathrm{mGy}$.

\subsection{Contrast and microcalcification visibility}

Figure 9 shows the CNR, as a function of the reconstructed slice thickness, both in phasecontrast imaging and in phase retrieval. The curve obtained for phase retrieval images takes values about one order of magnitude greater than that in phase-contrast imaging; in both cases the CNR increases as the slice thickness increases.

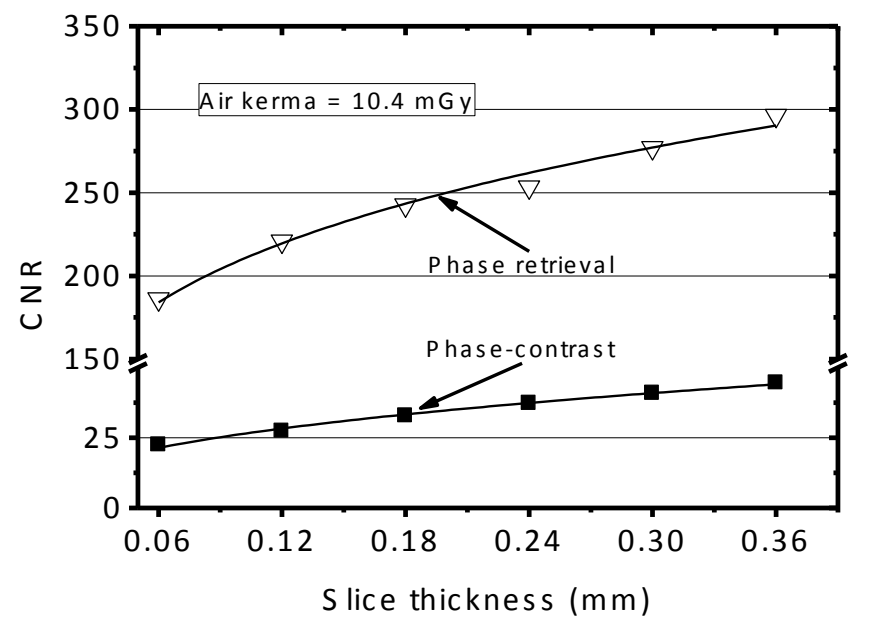

Figure 9: CNR between glandular and adipose tissue as a function of slice thickness in phase-contrast (closed square) and in phase retrieval (open triangles) imaging (Air kerma $=10.4 \mathrm{mGy}$, slice pixel size $=60 \times 60 \mu \mathrm{m}^{2}$ ). The continuous lines show a power law fit to the data points.

Figure 10 shows ROIs in CT slices (voxel size $=60 \times 60 \times 360 \mu \mathrm{m}^{3}$ ) which include the masses of the mammography test object. The CNR between masses and background, evaluated for the mass with a diameter of $3.16 \mathrm{~mm}$, was 45 in the phase retrieval images (fig. 10b) and less than 5 in the images obtained in phase-contrast imaging (fig. 10a). The high CNR in phase retrieval allows to make visible all the six masses with diameter ranging from 0.90 to $3.16 \mathrm{~mm}$ (fig. 10b), at variance with phase-contrast CT slices (fig. 10a). The dead spaces between adjacent detector blocks in the eight- 
units detector cause ring-like artefacts in reconstructed slices and reduces lesions visibility (e.g. lesion 6 in fig. 10b).

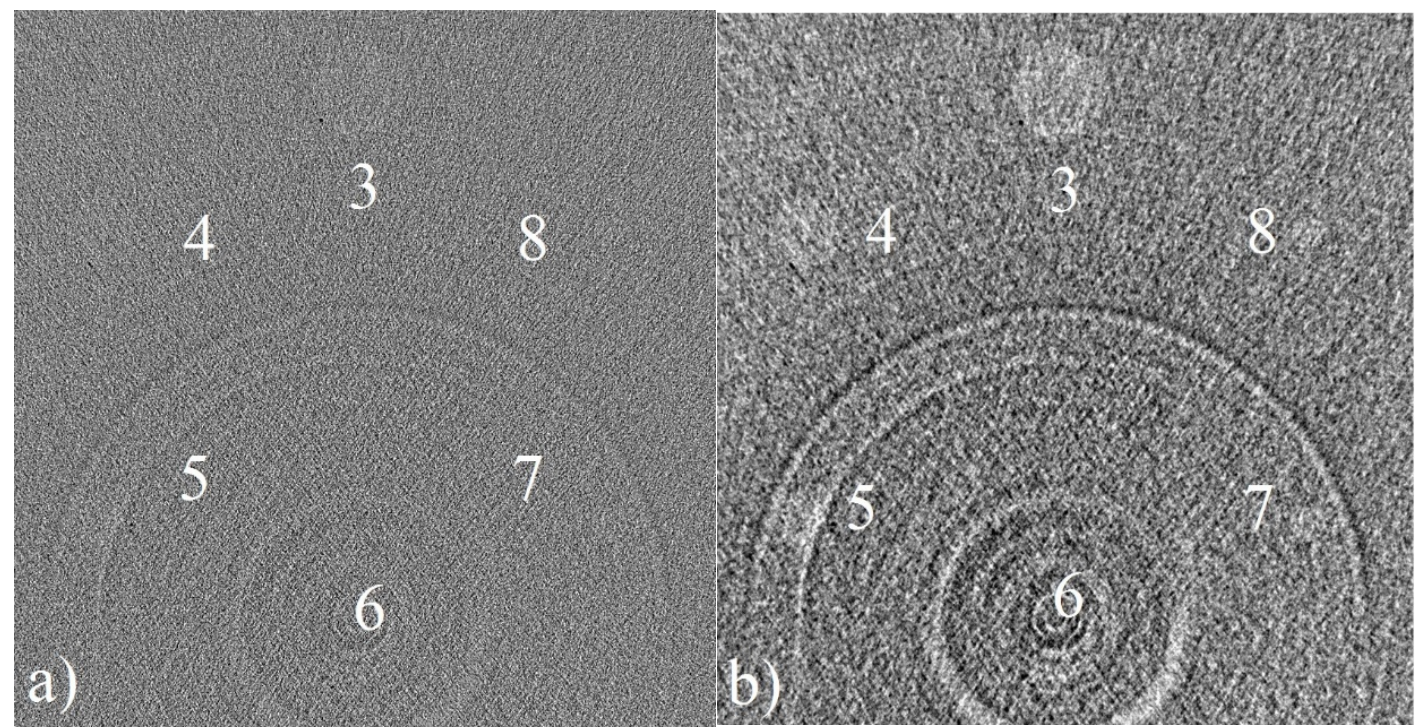

Figure 10: $75 \%$ glandular hemispheric masses with radius from 3.16 to $0.90 \mathrm{~mm}$ (labels 3-7, respectively), in phasecontrast (a) and with phase retrieval (b). Voxel size $=60 \times 60 \times 360 \mu \mathrm{m}^{3} ; 720$ projections; air kerma $=10.4 \mathrm{mGy}$.

Figure 11 shows images of two microcalcification clusters each represented by five $\mathrm{CaCO}_{3}$ specks, with diameter of $160 \mu \mathrm{m}$ and $130 \mu \mathrm{m}$, respectively. In spite of the drastic reduction of the system spatial resolution caused by the Paganing filter, the microcalcification clusters embodied in the mammographic CIRS phantom are visible both in phase-contrast (fig. 11a) and in phase retrieval (fig. 11b) reconstructed slices (voxel size of $60 \times 60 \times 120 \mu \mathrm{m}^{3}$ ). The black halos around the microcalcifications could be ascribed either to phase effects or to reconstruction artefacts. Furthermore, such an artefact can be ascribed to a manufacturing imperfection.
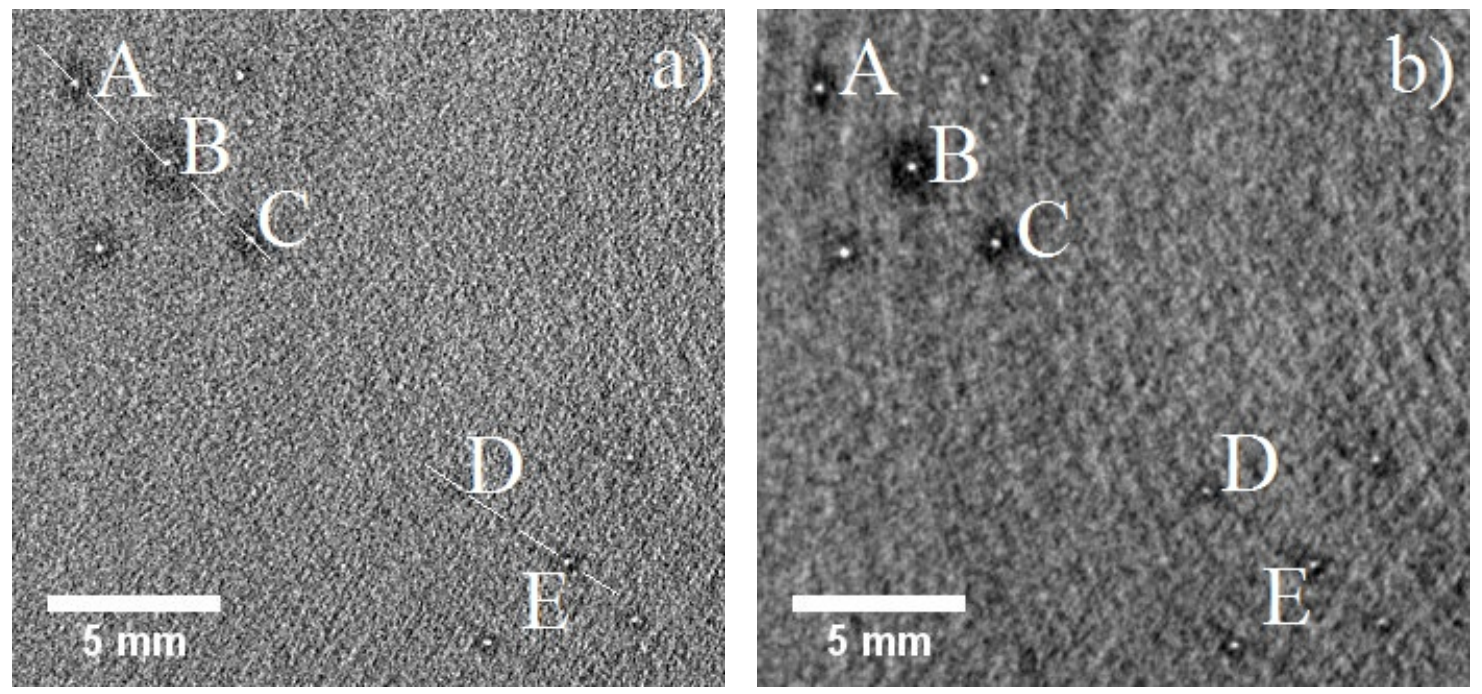
Figure 11: Microcalcification clusters with $\mathrm{CaCO}_{3}$ specks of $160 \mu \mathrm{m}$ and $130 \mu \mathrm{m}$ in phase-contrast (a) and in phaseretrieval (b) imaging. Voxel size $=60 \times 60 \times 120 \mu \mathrm{m}^{3} ; 720$ projections; air kerma $=10.4 \mathrm{mGy}$.

Figure 12 shows profiles over three of the five $160-\mu \mathrm{m}$ microcalcifications labeled in fig. 11 as $\mathrm{A}, \mathrm{B}$ and $\mathrm{C}$, in phase-contrast (fig. 12a) and in phase retrieval (fig. 12b), respectively. A Gaussian fit of the microcalcification profiles was performed, and then the FWHMs were evaluated from the fitting curves. The FWHMs evaluated for the $160-\mu \mathrm{m}$ microcalcification profiles are 150 $\mu \mathrm{m}$ and $212 \mu \mathrm{m}$, respectively in phase-contrast and in phase retrieval. Profiles over the microcalcifications of $130-\mu \mathrm{m}$ size labeled with $\mathrm{D}$ and $\mathrm{E}$ in fig. 11 are shown in fig. 13. In this case, the FWHM is $141 \mu \mathrm{m}$ in phase-contrast (fig. 13a) and $207 \mu \mathrm{m}$ for the images obtained after phase retrieval (fig. 13b).
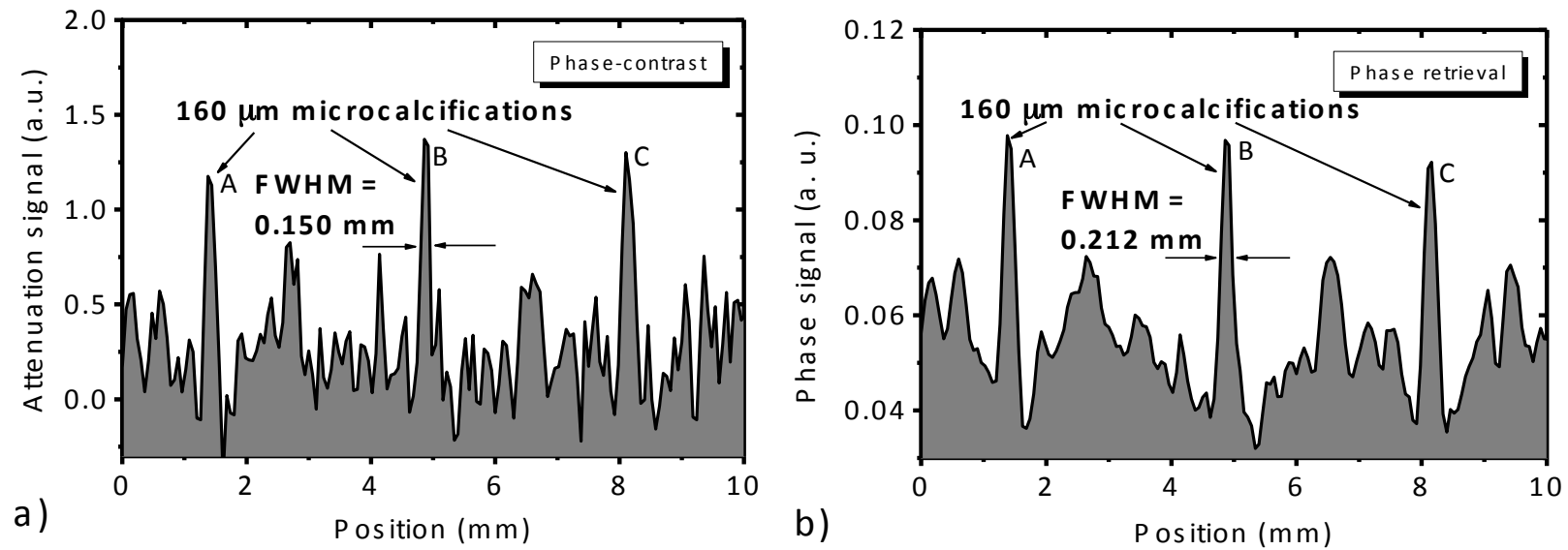

Figure 12: Profile over the $160-\mu \mathrm{m}$ microcalcification cluster (dashed line in fig 11a), labeled in fig. 11 with A, B and $\mathrm{C}$, in phase-contrast imaging, (a) and with phase-retrieval (b). Voxel size $=60 \times 60 \times 120 \mu \mathrm{m}^{3}$; air kerma $=10.4 \mathrm{mGy} ; 720$ projections.

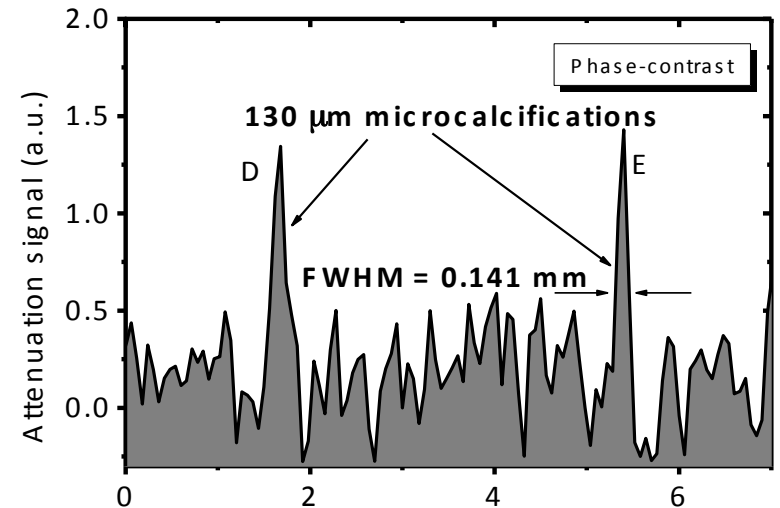

a)

Position $(\mathrm{mm})$

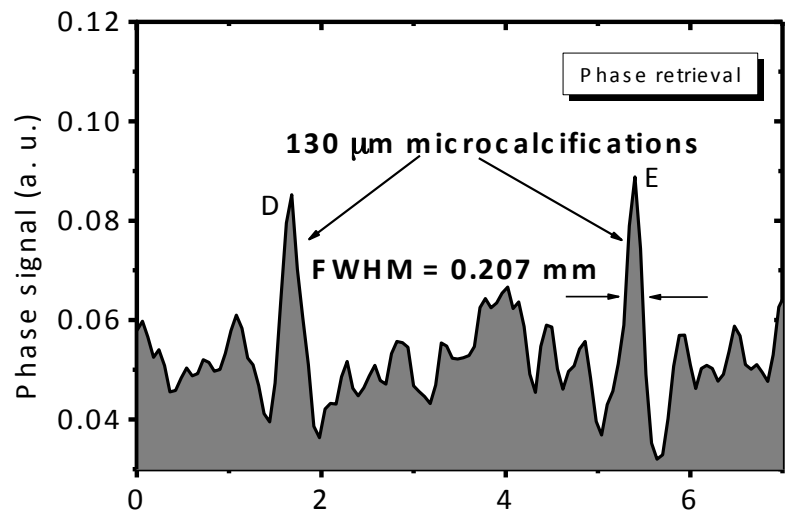

b)

$$
\text { Position }(\mathrm{mm})
$$

Figure 13: Profile over the $130-\mu \mathrm{m}$ microcalcification cluster (dashed line in fig 11a), labeled in fig. 11 with D and E, in phase-contrast imaging (a) and with phase-retrieval. Voxel size $=60 \times 60 \times 120 \mu \mathrm{m}^{3}$; air kerma at isocenter $=10.4$ mGy; 720 projections.

Table 1 reports the values of the global figure of merit $Q$ for phase-contrast imaging and for images obtained with the phase retrieval process. Such an index was evaluated for a voxel size of 
$(60 \mu \mathrm{m})^{3}$ as well as for a voxel size of $(120 \mu \mathrm{m})^{3}$. The $Q$ values indicate weak dependence of the phase-contrast image quality on the voxel size where the noise reduction balances the reduction of the spatial resolution; on the other hand, phase retrieval images with voxel size of $(60 \mu \mathrm{m})^{3}$ present a $Q$ index about 2.5 times higher than for a voxel size of $(120 \mu \mathrm{m})^{3}$. Indeed, in this case, increasing the voxel size reduces the system spatial resolution, but determines a weak influence on image noise. Moreover, for a voxel size of $(60 \mu \mathrm{m})^{3}$, application of the phase retrieval algorithm to phasecontrast images increases $Q$ by about 1.8 times. Hence, in the trade-off between system noise and system spatial resolution, this global figure of merit shows that phase retrieval may offer higher system imaging performance than phase-contrast images.

Table 1. Global figure of merit $Q$ (eq. 3) evaluated for phase-contrast and phase retrieval imaging with two different reconstruction voxel sizes.

\begin{tabular}{|c|c|c|}
\hline & \multicolumn{2}{|c|}{ Voxel size } \\
\hline & $60 \times 60 \times 60 \mu \mathrm{m}^{3}$ & $120 \times 120 \times 120 \mu \mathrm{m}^{3}$ \\
\hline Phase-contrast & 1523 & 1513 \\
\hline Phase retrieval & 2696 & 1084 \\
\hline
\end{tabular}

\section{Discussion}

\subsection{Image quality}

In this work, the authors evaluated the imaging performance of the SYRMA-CT system for in vivo breast imaging with monochromatic SR in terms of spatial resolution, noise, CNR and detail visibility, by analysing CT images of test objects, also containing simulated breast tissue lesions and microcalcification clusters.

The combination of fine pixel pitch, high resolution X-ray detector embodied in the SYRMACT scanner, the high-spatial coherence monochromatic X-ray beam and the large distance between scanned object and detector plane (about 2 meters), permitted to detect phase-contrast effects produced by X-ray beam diffraction in free-space propagation based imaging. No phase-contrast effects were present in CT slices of a breast specimen in a previous study at ELETTRA with the same geometry: this was attributed to the larger pixel pitch $(200 \mu \mathrm{m})$ of the detector used in that previous study, which hindered the visibility of the signal overshoot and undershoot at object edges [17]. In this work, with a $60-\mu \mathrm{m}$ pitch detector, reconstruction voxel size of $60 \mu \mathrm{m}$ and $120 \mu \mathrm{m}$ side preserved the phase-contrast information. In previous investigations at ELETTRA with monochromatic SR film-screen mammography, propagation-based phase-contrast imaging showed higher contrast detail visibility than conventional mammography at a comparable or lower dose [34]. 
In this new SR setup at ELETTRA, the fine pitch featured by the single-photon counting detector determined a spatial resolution as high as $6.7 \mathrm{~mm}^{-1}$ at $10 \% \mathrm{MTF}$, in slices with voxel size of $(60 \mu \mathrm{m})^{3}$ reconstructed via FBP algorithm and without phase retrieval (phase-contrast CT images). This system spatial resolution is significantly higher than that of polychromatic cone-beam BCT scanners [2], and compares well with the $5.6 \mathrm{~mm}^{-1}$ limiting frequency of the helical BCT scanner developed at University of Erlangen equipped with a high-resolution single-photon counting detector [35]. The spatial resolution limit of the SYRMA-CT setup reduced to $2.5 \mathrm{~mm}^{-1}$ when a Paganin filter was applied on the projections, in the application of the phase retrieval algorithm.

The edge enhancement effects seen in phase-contrast images of test objects increased the MTF curve to values higher than its zero-frequency value (fig. 6a); Honda et al [33], in propagation-based phase-contrast mammography, related a similar effect seeen in their images to an improvement of the edge sharpness of the object in projected images due to the phase effects.

The Paganin filter reduced drastically the image noise: the NNPS curves for phase-contrast imaging had a maximum value several times greater than those in phase retrieval images. The voxel size had weak influence on noise in images obtained with phase retrieval. The NNPS curves in phase-contrast imaging reached their maximum value at about $3 \mathrm{~mm}^{-1}$; on the other hand, the curves in phase retrieval imaging had their maximum at $1 \mathrm{~mm}^{-1}$, balancing in part the reduction of the system spatial resolution due to the Paganin filter. With the phase retrieval algorithm here adopted, the filter smoothing produced a FWHM size of the microcalcifications slightly higher than the actual size, but reduced the background signal fluctuation and preserved microcalcification visibility. In the proposed phantom study, $\mathrm{CaCO}_{3}$ microcalcifications with a diameter down to 130 $\mu \mathrm{m}$ were visibile.

In this work, the CNR in phase-contrast imaging was several times lower than that obtained with phase retrieval. The soft masses embodied in the mammographic test object were detected with high CNR when the phase retrieval process was applied to the projections. Correspondingly, for a CT slice thickness of $360 \mu \mathrm{m}$ and a slice pixel of $(60 \mu \mathrm{m})^{2}$, raw phase-contrast CT imaging produced a significantly lower masses visibility (CNR about six time lower).

A global figure of merit was evaluated, which takes into account spatial resolution and noise in CT slices. The analysis of this $Q$-index showed that in the trade-off between spatial resolution and noise in propagation-based phase-contrast imaging, the phase retrieval processing (with voxel size of $\left.(60 \mu \mathrm{m})^{3}\right)$ produced higher imaging performance than raw (i.e. without phase retrieval) phase-contrast imaging. The voxel size did not change significantly the $Q$-index in phase-contrast imaging, where reduction in the image noise balanced the reduction in the system spatial resolution. 
On the other hand, increasing the voxel size from $(60 \mu \mathrm{m})^{3}$ to $(120 \mu \mathrm{m})^{3}$ did not significantly reduce the noise in phase retrieval images but a reduction of the limiting spatial resolution from $2.5 \mathrm{~mm}^{-1}$ to $1.7 \mathrm{~mm}^{-1}$ was observed, with the global figure of merit reducing by as much as 2.5 times.

\subsection{Dose considerations}

In SYRMA-CT setup, discussed in ref [18], in order to image a whole breast with a monochromatic thin laminar beam, consecutive slices should be acquired (sequential CT). After the first 180 deg scan, the patient support is translated vertically by a step corresponding to the beam height (about $3 \mathrm{~mm}$ ) and a successive rotational (circular orbit) scan can be performed. The methods for the evaluation of the delivered dose in the SYRMA-CT configuration have been presented and discussed in refs. [21,36]. The imaging dose to the "glandular tissue" of the breast phantom used in this work was less than $4.2 \mathrm{mGy}$ [21]. The MGD to the breast in the case of whole breast SR-BCT irradiation (breast diameter $=12 \mathrm{~cm}$, breast height $=9 \mathrm{~cm}$, glandular fraction $=$ $50 \%$ ) with an air kerma at isocenter of $10.4 \mathrm{mGy}$, was estimated at $7.3 \mathrm{mGy}$ [21]. Such a glandular dose would be higher than, but comparable to, the one used in a two-view mammography exam: for a 12-cm diameter uncompressed 50/50 breast, which corresponds to a 3.2-cm thick compressed breast according to the determinations of Boone [37], the MGD for two-view mammography is approximately $2.5 \mathrm{mGy}$ (data from fig. 4 in ref. [37]). An MGD of $7.3 \mathrm{mGy}$ is comparable to that used for diagnostic (non screening) mammography exams, where the MGD could be between 6 and $36 \mathrm{mGy}$ (mean $=16.9 \mathrm{mGy}$, standard deviation $=6.9 \mathrm{mGy})$ [38]. An estimated MGD of $7.3 \mathrm{mGy}$ is also lower than that evaluated for the Koning Corp. commercial BCT scanner - recently approved by FDA in USA for diagnostic imaging of the breast in conjunction with two-view mammography - which released to the breast a mean glandular dose ranging between $14.0 \mathrm{mGy}$ and $17.5 \mathrm{mGy}$ [39].

\section{Conclusions}

The authors evaluated the imaging performance of the SYRMA-CT system dedicated to the breast, at the ELETTRA SR facility, in terms of MTF curves, normalized NPS curves, microcalcifications visibility and soft masses visibility, using test objects simulating the attenuation properties of breast tissues.

The system spatial resolution reached $6.7 \mathrm{~mm}^{-1}$ prior to the application of the phase retrieval algorithm to the projections, while the Paganin filter for phase retrieval caused a spatial resolution reduction down to a frequency of $2.5 \mathrm{~mm}^{-1}$ at $10 \% \mathrm{MTF}$. On the other hand, the phase retrieval following the Paganin algorithm caused a related increasing in detail CNR by one order of 
magnitude. The visibility of simulated soft masses was improved via this algorithm, and the CNR of masses was more than six times lower in the images prior to phase retrieval. Clusters of simulated microcalcifications with a diameter down to $130 \mu \mathrm{m}$ were visible both before and after phase retrieval. The air kerma at isocenter here adopted for imaging would produce MGD values comparable to that used in two-view mammography in case of whole breast irradiation for a $12-\mathrm{cm}$ diameter breast. The evaluation of a global figure of merit, which took in account spatial resolution and noise in CT slices, indicated that the phase retrieval offers superior imaging performance than phase-contrast, in monochromatic SR imaging of breast phantoms.

\section{Acknowledgment}

The authors thank all colleagues of the SYRMA-CT collaboration. This work has been carried out in the framework of the SYRMA-CT collaboration funded by INFN, Italy. The authors have no conflict of interest related to this study.

\section{References}

[1] BCSC 2009 Website: http://breastscreening.cancer.gov/statistics/performance/screening/2009/perf_age.html. [accessed 07.03.2015].

[2] Sarno A, Mettivier G, Russo P. Dedicated breast computed tomography: basic aspects. Med Phys 2015; 42:27862804.

[3] Castelli E, Tonutti M, Arfelli F, Longo R, Quaia E, Rigon L et al. Mammography with synchrotron radiation: first clinical experience with Phase-Detection technique. Radiology 2011; 250:684-94.

[4] Quai E, Longo R, Zanconati F, Jaconelli G, Tonutti M, Abrami A et al. First application of computed radiology to mammography with synchrotron radiation. Radiol Med 2013; 118:89-100.

[5] Longo R, Tonutti M, Rigon L, Arfelli F, Dreossi D, Quai E et al. Clinical study in phase- contrast mammography: image-quality analysis. Phil Trans R Soc A 2014; 372:1-8.

[6] Fiedler S, Bravin A, Keyriläinen J, Fernández M, Suortti P, Thomlinson W et al. Imaging lobular breast carcinoma: comparison of synchrotron radiation DEI-CT technique with clinical CT, mammography and histology. Phys Med Biol 2004; 49:175-188.

[7] Bravin A, Coan P, Suortti P. X-ray phase-contrast imaging: from pre-clinical applications towards clinics. Phys Med Biol 2013; 58:R1-R35.

[8] Keyriläinen J, Fernández M, Fiedler S, Bravin A, Lindsberg MLK, Virkkunen P et al. Visualisation of calcifications and thin collagen strands in human breast tumour specimens by the diffraction-enhanced imaging technique: a comparison with conventional mammography and histology. Eur J Radiol 2005; 53:226-237.

[9] Keyriläinen J, Fernaández M, Lindsberg MLK, Virkkunen P, Leidenius M, von Smitten K, et al. Toward HighContrast Breast CT at Low Radiation Dose. Radiology 2008; 249:321-327.

[10] Momose A, Takeda T, Itai Y, Hirano K. Phase-contrast X-ray computed tomography for observing biological soft tissues. Nat Med 1996; 2:473-75. 
[11] Sztrókay A, Herzen J, Auweter SD, Liebhardt S, Mayr D, Willner M et al. Assessment of grating-based X-ray phase-contrast CT for differentiation of invasive ductal carcinoma and ductal carcinoma in situ in an experimental ex vivo set-up. Eur Radiol 2013; 23:381-87.

[12] Takeda T, Momose A, Ueno E, Itai Y. Phase-contrast X-ray CT image of breast tumor. J Synchrotron Radiat $1998 ; 5: 1133-35$.

[13] Takeda T, Momose A, Hirano K, Haraoka S, Watanabe T, Itai Y. Human Carcinoma: Early Experience with Phase-Contrast X-ray CT with Synchrotron Radiation-Comparative Specimen Study with Optical Microscopy. Radiology 2000; 214:298-301.

[14] Zhao Y, Brun E, Coan P, Huang Z, Sztrókay A, Diemoz PC et al. High-resolution, low-dose phase contrast X-ray tomography for 3D diagnosis of human breast cancers. Proc Nat Acad Sci USA 2012; 109:18290-94.

[15] David C, Weitkamp T, Pfeiffer F, Diaz A, Bruder J, Rohbeck T et al. Hard X-ray phase imaging and tomography using a grating interferometer. Spectr Acta B 2007; 62:626-630.

[16] Cai W and Ning R. Dose efficiency consideration for volume-of-interest breast imaging using X-ray differential phase-contrast CT. Proc SPIE 7258, Med. Imag. 2009: Physics of Medical Imaging, 72584D

[17] Pani S, Longo R, Dreossi D, Montanari F, Olivo A, Arfelli F et al. Breast tomography with synchrotron radiation: preliminary results. Phys Med Biol 2004; 49:1739-1754.

[18] Longo R, Arfelli F, Bellazzini R, Bottigli U, Brez A, Brun F et al. Towards breast tomography with synchrotron radiation at Elettra: first images. Phys Med Biol 2016; 61: 1634-1649.

[19] Pacilè S, Brun F, Dullin C, Nesterets YI, Dreossi D, Mohammadi S et al. Clinical application of low-dose phase contrast breast CT: methods for the optimization of the reconstruction workflow. Biomed Opt Express 2015; 6:3099-3112.

[20] Nesterets YI, Gureyev TE, Mayo SC, Stevenson AW, Thompson D, Brown JMC et al. A feasibility study of Xray phase-contrast mammographic tomography at the Imaging and Medical beamline of the Australian Synchrotron. J Synchrotron Rad 2015; 22:1509-1523.

[21] Mettivier G, Fedon C, Di Lillo F, Longo R, Sarno A, Tromba G, Russo P. Glandular dose in synchrotron radiation breast computed tomography. Phys Med Biol 2015;61:569-587.

[21bis] Mittone A, Bravin A and Coan P. Radiation dose in breast CT imaging with monochromatic x-rays: simulation study of the influence of energy, composition and thickness. Phys Med Biol 2014; 59:2199-2217.

[22] Kuttig JD, Steiding C, Kolditz D, Hupfer M, Karolczak M, Kalender WA. Comparative investigation of the detective quantum efficiency of direct and indirect conversion detector technologies in dedicated breast CT. Phys Med 2015; 31:406-416.

[23] Lopez FC, Rigon L, Fardin L, Arfelli F, Bergamaschi A, Dreossi D et al. Comparator threshold settings and the effective pixel width of the PICASSO detector. J Instrum 2014; 9-C05056:1-8.

[24] Bellazzini R, Spandre G, Brez A, Minuti M, Pinchera M, Mozzo P. Chromatic X-ray imaging with a fine pitch CdTe sensor coupled to a large area photon counting pixel ASIC. J Instrum 2013; 8-C2028:1-8.

[25] Paganin D, Mayo SC, Gureyev TE, Miller PR, Wilkins SW. Simultaneous phase and amplitude extraction from a single defocused image of a homogeneous object. J Microsc 2002; 206:33-40.

[26] Xop 2.3 - website: http://www.esrf.eu/Instrumentation/software/data-analysis/xop2.4. [accessed 15.10.2015].

[27] Boone JM. Determination of the presampled MTF in computed tomography. Med Phys 2001; 28:356-360.

[28] Kwan ALC, Boone JM, Yang K, Huang SY. Evaluation of the spatial resolution characteristics of a cone-beam breast CT scanner. Med Phys 2007; 34:275-281. 
[29] Mettivier G and Russo P. Measurement of the MTF of a cone-beam breast computed tomography laboratory scanner. IEEE Trans Nucl Sci 2011; 58:703-713.

[30] CSIRO - website: https://ts-imaging.net/Services/Simple/ICUtilXdata.aspx. [accessed 15.10.2015].

[31] Yang K, Kwan ALC, Huang SY, Packard NJ, Boone JM. Noise power properties of a cone-beam CT system for breast cancer detection. Med Phys 2008; 35:5317-5327.

[32] Kalender WA. Computed tomography. 2nd rev. Ed. Publicis Corporate Publishing 2005.

[33] Honda C, Ohara H, Gido T. Image qualities of phase-contrast mammography. Digital Mammography 8th International Workshop, IWDM 2006 Manchester, UK, June 18-21, 2006 Proceedings 4046:281-288.

[34] Castelli E, Arfelli F, Dreossi D, Longo R, Rokvic T, Cova MA et al. Clinical mammography at the SYRMEP beam line. Nucl Instrum Meth A 2007; 572:237-240.

[35] Steiding C, Kolditz D, Roessler AC, Kalender WA. Quantitative assessment of the spatial dependence of nonstationary high-contrast spatial resolution, low-contrast detectability, and noise behavior in 3D imaging of the breast. 20th European Congress of Radiology (ECR) 2014, March 6 - 10, Vienna, Austria.

[36] Fedon C, Longo F, Mettivier G, Longo R. GEANT4 for breast dosimetry: parameters optimization study. Phys Med Biol 2015; 60: N311-N323.

[37] Boone JM, Kwan AL, Seibert JA, Shah N, Lindfors KK and Nelson TR. Technique factors and their relationship to radiation dose in pendant geometry breast CT. Med Phys 2005; 32:3767-3776.

[38] O’Connell A and O’Connor DK. Dedicated Cone-beam Breast Computed Tomography and Diagnostic Mammography: Comparison of Radiation Dose, Patient Comfort, And Qualitative Review of Imaging Findings in BI-RADS 4 and 5 Lesions. J Cli Imaging Sci 2012; 2:1-8.

[39] Sechopoulos I, Feng SSJ and D’Orsi CJ. Dosimetric characterization of a dedicated breast computed tomography clinical prototype. Med Phys 2010; 37:4110-20.

[40] Abrami A , Arfelli F, Barroso RC, Bergamaschi A, Bille` F et al. Medical applications of synchrotron radiation at the SYRMEP beamline of ELETTRA. Nucl Instr Meth A 2005; 548: 221-227 